\title{
Architecture Research on Citrus Planting Expert System
}

\author{
Guibo Ni \\ School of Zhonghuan Information College Tianjin University of Technology
}

Keywords: Expert system, Architecture, Knowledge base.

\begin{abstract}
Comparatively speaking, the expert system is a much rapid development direction in the computer control field recently. Combine expert system and agriculture together to let computers guide the application and development of agriculture and promote agricultural informationization and high-tech. This paper presents open and flexible citrus planting expert system architecture on the basis of comprehensive analysis software architecture and citrus planting state.
\end{abstract}

\section{Introduction}

A sustainable development agriculture can decided the agriculture science development including new agricultural production technologies, agricultural biotechnology and intelligent agricultural information technology. Since the middle of the last century 80 's, an agriculture intelligent information technology, being a hot topic of computer application research in domestic and overseas, has been widely applied and developed in the field of agricultural production. What demands apply information technology to agriculture successfully depends on the information technology hardware level and software, the most important element at the same time, which can meet characteristics of agricultural production and the agricultural research technician practical application. To make citrus producers obtain scientific production management direction and provide reasonable auxiliary information to the relevant primary sector managers and agricultural technical personnel. This system absorbs others advantages and refers practical experience, learns from practical experience of experienced planter, wisdom of the leading authority experts in this field as well as the most sophisticated research results in the industry research area ${ }^{[1-3]}$.

\section{Software Architecture}

Software architecture (SA), an important branch of software engineering, attracts academics and industry interests from the date of birth dramatically. It is an intermediary for requirements specification and implementation, including a number of components, connector, as well as some of the constraints, which provide guidance for the organization and realization of the system blueprint. Specifically speaking, the functions of SA are:

SA provides ideological support for understanding large-scale software systems it is convenient for the reuse of components and system level it can illustrate the main system components as well as their relationships and constraints. It can clearly explain the variability of the system and verify the system goals with the deepening research in the field of software engineering; SA becomes indispensable and plays a critical role in the system design. Good software architecture is meaningful in all phases of the software life cycle. In the systems analysis phase, software architecture description can make the questions to be more abstract and help analysis and design researchers have a clearly understanding about the system, even more conscious recognition. In addition, it enables user developers form a unified understanding of the system function, and enhances communication and system improvement. In terms of system implementation, software architecture provides design decisions for the subsequent development, which has a special meaning for software quality control and maintenance of the organizational structure. Software architecture achieves the changes from code-level reuse to the design and process reuse ${ }^{[4-6]}$. 


\section{Citrus planting expert system architecture}

Citrus planting expert system architecture proposed in this paper shows in Figure 1.

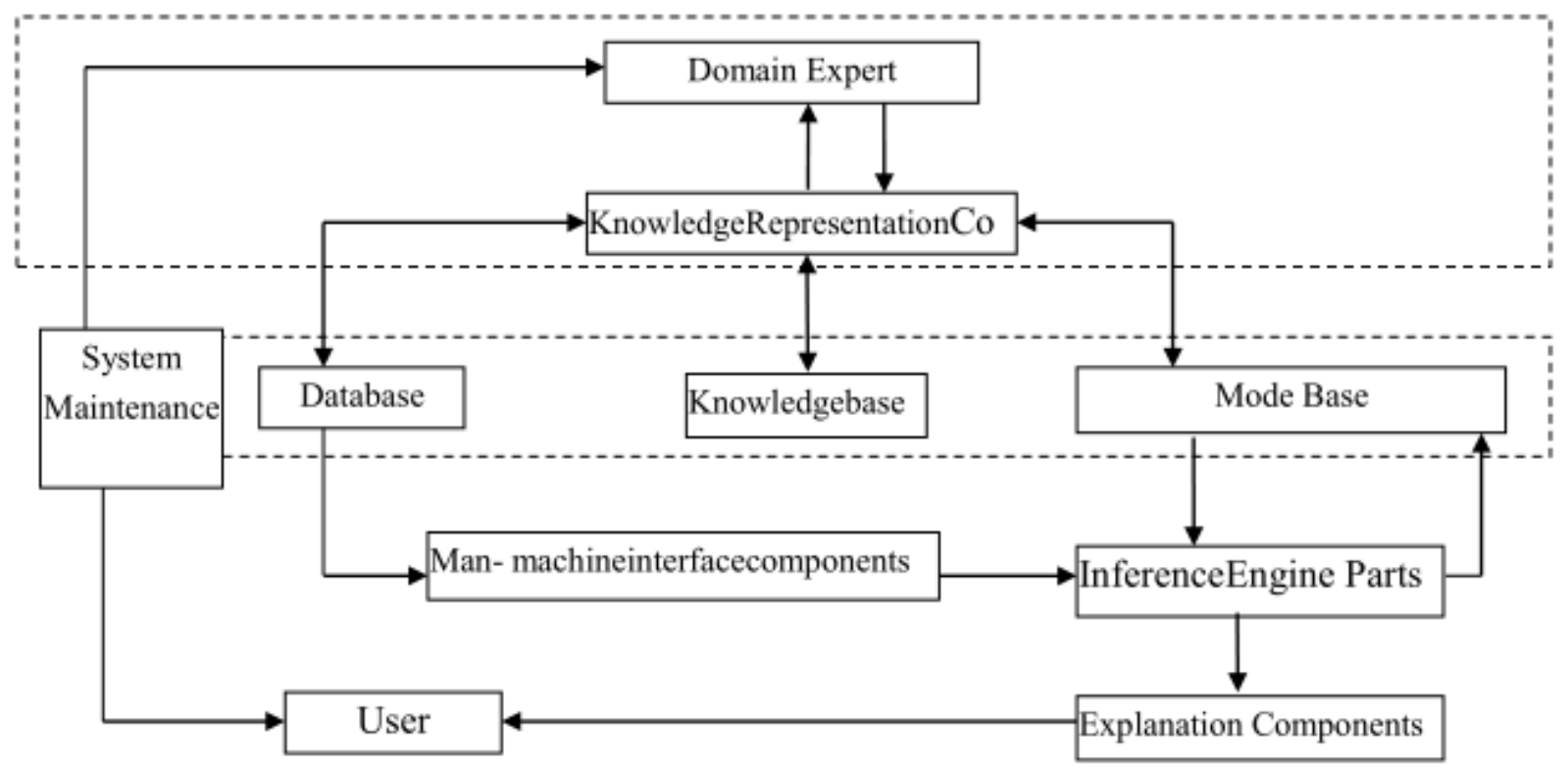

Figure 1 citrus planting expert system

On the basis of original citrus planting expert system, the system can be divided into three parts, including knowledge acquisition, database and expert system core components, and added the explained component which makes the expert system better. Contents in virtual box on the above, a kind of knowledge acquisition, is accumulated by the basic theory, experience and research achievements of agriculture experts at a long time, then inputted to the expert system and represented it in knowledge representation structures. The second virtual box is a database, including library, database and model base, which is used to store various kinds of knowledge abstracts. The rest parts are the core of expert systems, composed by the inference engine parts, components, human-machine interface components and system maintenance and so on.

Knowledge Acquisition. Knowledge acquisition is to acquire knowledge from experts or other specialized knowledge sources and the process or technology to transform to knowledge. The implementation of knowledge acquisition and establishment of knowledge system is overlapping. Generally, at the beginning of knowledge system construction, only necessary knowledge is got, and with the commissioning and operation of the system, new knowledge is gradually accumulated. When knowledge base is expanded and updated, the compatibility of old and new knowledge needed to be checked, in order to maintain the integrity of the knowledge base ${ }^{[7-8]}$.

Inference Engine. In general, performance and structure of inference engine is relevant with knowledge organization, and has nothing to do with the content of the knowledge, which is advantageous to relative independence between inference engine and knowledge base. When knowledge is changed, no need to modify the inference machine. However, search strategy of inference engine could not completely independent of the domain knowledge; otherwise the effectiveness will be reduced ${ }^{[9]}$.

Explanation Component. In order for a user to understand the conclusion of the system, explanation component increased in the system. It is the module designed in order to resolve the problems of interpretation. The interpretation strategy of this system is preset text, that is, to make an organization framework of interpretation to explain the problem by using a natural language, and insert it into the corresponding database. Explanatory information is generated when the results are presented to the users. 


\section{Conclusion}

This paper presents a new architecture of citrus planting expert system based on the integrated introduction about the software one, and increases the explanation components as well as makes a classification on the whole architecture, in order to make each function clear. However, the advantages of this architecture have not been digitally verified. Therefore, its optimum needs verifying.

\section{References}

[1] Yang Fuqing, Wang Qianxiang, Mei Hong, et al. Reuse-based software production technology [J]. Science in china (Series F), 2011, 44(1): 8-19.

[2] Raggl A, Slany W A. Reusable iterative optimization software library to solve combinatorial problems with approximate reasoning [J]. International Journal of Approximate Reasoning, 2013, 19(1/2): 161-191.

[3] NATO. Standard for Management of a Reusable software component library [EB/OL]. http://www.uml.org.cn/bzgf/component/nato_standards_vol_2.pdf.2011/2011-9-16.

[4] Jean-Guy Schneider, Components, Scripts, and Glue,A concept framework for software composition. PhD dlesis, University ofBern, Deutschland, Oct, 2012.

[5] Markus Lumpe, A g-Calculus Based Approach for software Composition, PhD thesis, University ofBern, Deutschland, Jan, 1999

[6] Medvidovic N, Taylor R.N. A Classification and Comparison Framework for Software Architecture Description Language. IEEE Transactions on Software Engineering, 2011, 26(1): 70-93.

[7] Oded Maimon Lior Rokach. Improving Supervised Learning by feature Decomposition. Foundations of Information and Knowledge Systems, 2012, (2):178-196.

[8] Bao Fu, Li Jin Liu.Detail feature recognition and decomposition in solid model. Computer-aided design, 2012, 34(5):405-414.

[9] Rafael Bidarra, Eelco van den Berg and Willem F. Bronsvoort. Collaborative modeling with features. In: The 21th Computers and information in engineering conference, 2011, 1(1): 535-545. 